\title{
Sur les traces de Liberté, Égalité, Fraternité d'Agnès Rosenstiehl
}

\section{Karine Meshoub-Manière}

\section{(2) OpenEdition}

1 Journals

Édition électronique

URL : http://journals.openedition.org/genesis/4080

DOI : 10.4000/genesis.4080

ISSN : 2268-1590

Éditeur :

Presses universitaires de Paris Sorbonne (PUPS), Société internationale de génétique artistique littéraire et scientifique (SIGALES)

Édition imprimée

Date de publication : 1 juin 2019

Pagination : 93-107

ISSN : 1167-5101

Référence électronique

Karine Meshoub-Manière, "Sur les traces de Liberté, Égalité, Fraternité d'Agnès Rosenstiehl », Genesis [En ligne], 48 | 2019, mis en ligne le 01 juin 2020, consulté le 24 janvier 2021. URL : http:// journals.openedition.org/genesis/4080 ; DOI : https://doi.org/10.4000/genesis.4080 


\title{
Sur les traces de Liberté, Égalité, Fraternité d'Agnès Rosenstiehl
}

\author{
Karine Meshoub-Manière
}

Q uand on parcourt l'œuvre d'Agnès Rosenstiehl, auteure et illustratrice, l'on ne peut qu'être frappé par la double orientation de son travail : d'une part, son goût pour la langue, les mots, le jeu avec les lettres, qui relève d'une «espèce de maniaquerie », selon les mots de l'auteure, comme en témoignent sa production en matière d'abécédaires ou de dictionnaires ${ }^{1}$ ainsi que toutes les traces dans ses œuvres de jeux de mots qui la rapprochent de l'OULIPO; et d'autre part, son souci de déconstruire les stéréotypes (souvent sexistes), en invitant son public à réfléchir, à travers la fiction, aux comportements à adopter face aux stéréotypes. Que l'on songe au personnage de Mimi Cracra qui a valu à Agnès Rosenstiehl sa notoriété auprès du grand public avec près de 250 épisodes réalisés, cette fillette de quatre ans pleine de vie, maligne et désobéissante, prête à explorer le monde, «qui s'amuse de tout, de tout ce qui l'entoure et qui invente, toute seule, à quoi jouer et comment jouer avec rien ${ }^{2} »$.

Si son œuvre est à destination de la jeunesse (comme le donnent à penser les maisons d'édition qui la publient), l'auteure avoue chercher à séduire, au-delà de l'enfant, l'adulte qui la lira pour lui-même ou pour des/ses enfants. En effet, si elle s'interroge sur ce qui peut toucher, faire rire ou réfléchir les jeunes lecteurs, Agnès Rosenstiehl espère interroger et embarquer dans son univers de valeurs et de poésie tout lecteur qui aura su garder une part de son enfance en lui, sa fraîcheur et son goût pour la surprise et l'inattendu. Le plaisir qu'elle prend à construire ses œuvres, la jubilation que lui procurent ses essais avec la langue, elle espère les susciter chez son lecteur, adulte comme enfant, de 7 à 77 ans. «J'ambitionne les lecteurs de Tintin», nous confie-t-elle. Une telle déclaration interroge sur la désignation même de «littérature de jeunesse » où la préposition « de» annonce le public visé.

Parmi la foisonnante production d'Agnès Rosenstiehl, nous souhaitons nous intéresser, dans le cadre de cette contribution, à la genèse de Liberté, Égalité, Fraternité3.

\section{Présentation de l'œuvre}

Cet album, à destination des 3-6 ans, a pour ambition de faire comprendre à son jeune public, les trois termes de la devise républicaine française, à travers des scènes de leur vie quotidienne, vécues par des enfants du même âge. Il comprend trois parties, correspondant chacune à l'un des termes de la devise. Une page bleue sépare les volets «Liberté» et «Égalité», une page rouge les volets «Égalité» et «Fraternité». L'ensemble de l'album est réalisé sur fond blanc, sur lequel se détache le texte, écrit en noir, au pinceau, comme en témoigne l'irrégularité du trait. Quand il s'agit de discours direct, le texte est alors placé dans un phylactère, de forme rectangulaire.

Pour ce qui est de l'illustration, les personnages sont tous dessinés de profil, c'est un trait caractéristique de l'auteure-illustratrice, qui se dit fascinée par l'antiquité égyptienne. Le dessin est épuré, minimaliste, une attention particulière est apportée à l'expression du visage : le contour des personnages est réalisé au pinceau, toujours en noir, les

1. À titre indicatif, nous signalons quelques œuvres centrées sur la langue, abécédaires ou dictionnaires

$A B C$, Éditions des Jumeaux, 1969;

Drôle d'alphabet ou les aventures d'une tarte aux pommes, Larousse, 1977 ;

Mon Premier Alphabet, Larousse, 1977 ; Lire c'est partir, 2005 ; L'Alphabet fou ou le livre des syllabes sibyllines, Larousse, 1978;

L'alphabet fait des histoires, illustrations de Pierre Gay, Gallimard, 1984 ; Lire c'est partir, 1998 ;

Le Larousse des tout-petits. Les Verbes, Larousse, 1986;

Le Larousse des tout-petits. Les Adjectifs, Larousse, 1987;

Le Larousse des tout-petits. Les Adverbes, Larousse, 1994;

Réédition des trois derniers en un seul volume en 2000.

2. Propos recueillis lors d'une émission de radio, «Mimi Cracra ou l'éternelle jeunesse», diffusée le 9 mai 2017 sur France Culture (Cinqfois, par Maylis Besserie).

3. Agnès Rosenstiehl, Liberté, Égalité, Fraternité, Paris, Le Seuil 1999. Réédition en 2016 aux Chemins de tr@verse, dans un format différent (plus grand), mais sans modification, ni du texte ni de l'illustration. 


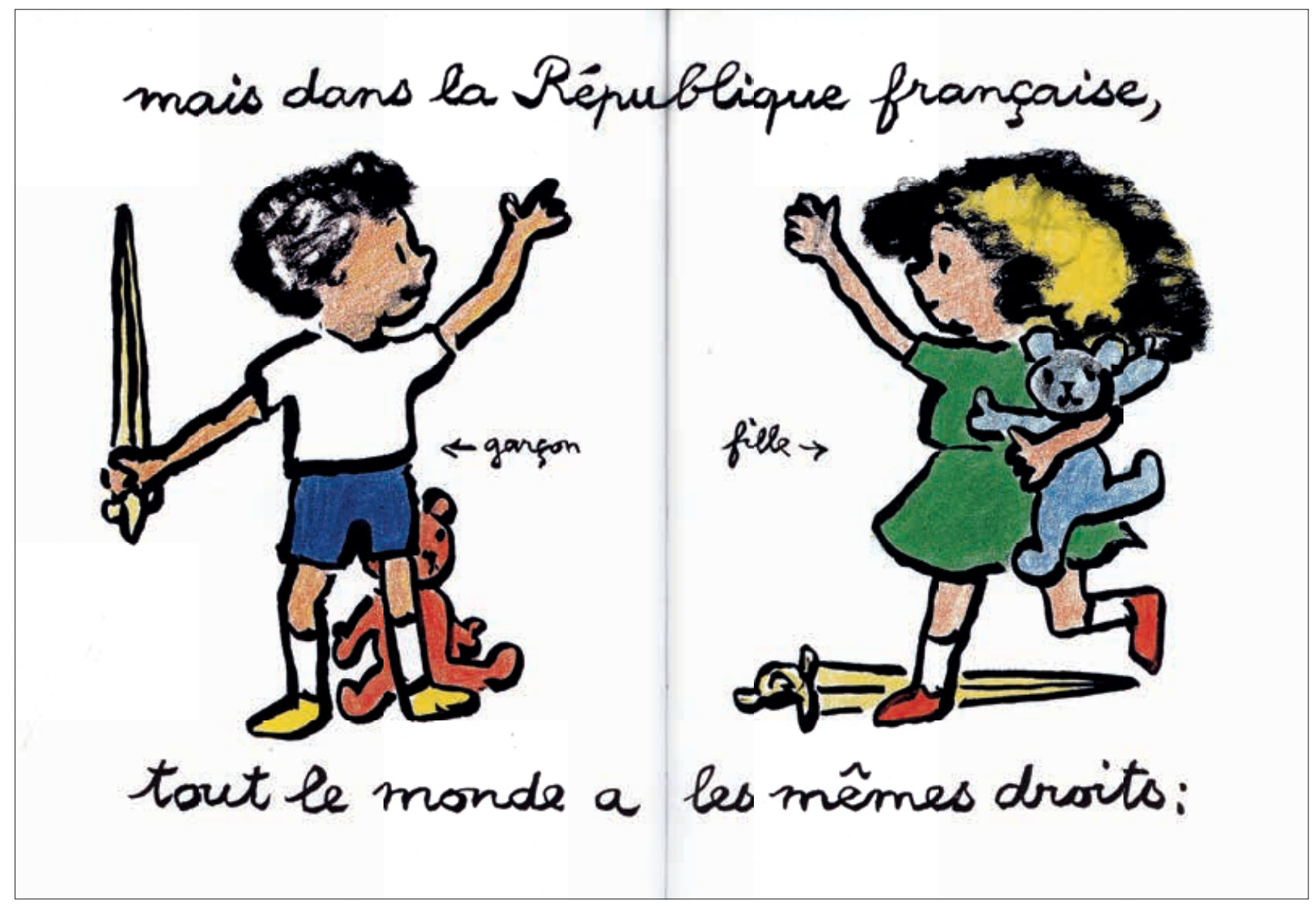

Fig. 1 : Double page (16-17) de la version éditée - Agnès Rosenstiehl, Liberté, Égalité, Fraternité, Neuville-sur-Saône, Éditions Chemins de Tr@ verse, 2016 -Format : 14,5 × 20 cm.

couleurs sont appliquées avec des pastels gras, de couleurs franches (principalement bleu, rouge, jaune, vert).

La répartition texte/illustration sur l'espace de la double page est variable, de même que l'attention portée à la pliure. Chacun des trois volets se compose de dix-sept doubles pages.

Dans le volet «Liberté», le personnage principal cherche à explorer l'étendue de sa liberté. Il se met donc en quête de ce qu'il pense avoir le droit de faire. À partir de là (p. 6), chaque double page fonctionne par deux, avec une séquentialité dans l'organisation texte/image : le personnage principal agit sans tenir compte des autres, ni de ce qui l'entoure en page de gauche, son attitude entraînant en page de droite une réaction émotionnelle de ses protagonistes, qui en page suivante opposent une réaction (action) à laquelle notre premier personnage réagit à son tour, en page de droite. Ce diptyque, reposant sur ce que provoque chez les autres le comportement égoïste de notre personnage principal, se poursuit jusqu'à l'antépénultième double page avec une répartition texte/image à gauche et à droite variable.

Dans le volet «Égalité », l'auteure cherche à faire comprendre au jeune lecteur que derrière les différences de chacun, qu'elles soient innées, culturelles ou sociales, il y a une égalité de droits. L'auteure choisit de poser une première différence fondamentale, naturelle entre les êtres humains, la couleur de peau (pages 4-5 à 14-15), avant de rappeler cette égalité des droits au-delà des différences, auxquelles s'ajoute la différence fille-garçon, problématique chère à l'auteure (double page 16-17, fig. 1) ; s'ensuit alors une déclinaison de droits valables pour tous (pages 18-27), dont le dernier, celui de manger, réunit tout le monde autour d'une même table.

Si la première double page (pages 2-3) énonce l'appartenance de «tous les bébés qui naissent» à la même «famille des êtres humains », l'avant-dernière (pages 30-31) qui lui répond («Dans la famille des êtres humains, / on n'est pas des bêtes ») interpelle le lecteur : pas question pour l'auteure d'établir ici une hiérarchie entre l'homme et l'animal où le premier l'emporterait sur le second. Si le chat, sur la double page précédente (28-29, cf. infra fig. 10), se plie à l'ordre de la nature en chassant une souris (où derrière le respect du principe de la chaîne alimentaire, on pourrait être tenté de lire le principe de la loi du plus fort), l'Homme ne peut agir ainsi et obéir au même principe de réalité : notre rapport à l'autre se situe ailleurs, sur le terrain des droits, dans l'intelligence de l'Autre et de Soi. 
Dans le volet «Fraternité», il s'agit de faire découvrir aux enfants le principe de fraternité qui repose sur le partage et l'acceptation de l'autre : l'univers choisi par l'auteure est ici celui du jeu. Les quatre premières doubles pages présentent des enfants qui jouent chacun dans leur coin. Très vite, l'ennui que suscite le fait de jouer seul et avec peu de jouets incite les enfants à mettre en commun leurs jouets (double page 10-11). S'ouvre alors un temps d'exploration collective du jeu (pages 12-17) avant que n'arrive l'heure du goûter (double page 18-19), qui devient, à son tour, l'occasion de faire l'expérience du partage : comme l'un des enfants n'a rien à manger (double page 20-21) les autres lui proposent de partager ce qu'ils ont (pages 22-25). Une fois les troupes rassasiées, grâce à l'arrivée d'un nouveau compagnon, ballon au pied (double page 26-27), place de nouveau au jeu collectif et au plaisir qu'il suscite (pages 28-31).

Au terme de cette présentation de l'œuvre, ce qui apparaît au lecteur adulte, c'est une certaine forme de simplicité, pour chaque partie du triptyque, dans la structure du texte et dans l'articulation texte/image pour un message qui, lui, n'a rien d'évident pour de jeunes enfants, en raison de son degré d'abstraction et de complexité. C'est précisément cette simplicité apparente que nous souhaiterions interroger et analyser à travers l'exploration de quelques documents de genèse : comment l'auteure construit-elle, dans le texte et dans l'image, cette simplicité qui fait que l'œuvre opère sur son lecteur?

\section{Présentation du dossier génétique}

Notre analyse génétique ne portera que sur les deux premiers volets de ce triptyque, «Liberté» et «Égalité » : le premier parce qu'il permet d'approcher le mode d'écriture de l'auteure par essais successifs, allant d'une phase de recherche, d'exploration (qui consiste selon ses propres mots à «rester dans les méandres de la Seine»), à une phase d'approfondissement qui ne cesse que lorsqu' arrive la date de remise du manuscrit à l'éditeur; le second parce qu'il permet de mesurer le chemin parcouru entre les différentes phases de recherche et la version éditée ${ }^{4}$; et dans le même temps, il permet d'interroger ce qui se joue dans l'élaboration d'un texte pour la jeunesse. Nous laissons volontairement de côté le volet «Fraternité», car l'auteur $\mathrm{y}$ procède de la même façon que dans le premier.

Ce dossier génétique, conservé à l'IMEC (Institut Mémoires de l'édition contemporaine)5, contient l'ensemble des étapes de l'élaboration de l'œuvre jusqu'à son édition pour chacun des trois volets : un canevas de l'œuvre, des pistes de thèmes à aborder, un ou plusieurs chemins de fer (comprenant l'ensemble des doubles pages de l'album, matérialisées par seize cases divisées chacune en deux, sur lesquelles l'auteure organise la répartition texte/illustrations), différentes versions 6 d'un même texte avec différents états de l'illustration, de la simple esquisse à une forme plus élaborée; des maquettes pour vérification avant impression; quatre petits carnets (de format variable) comprenant à la fois des essais où l'auteure explore des possibles textuels et iconiques, mais aussi où elle reprend les essais réalisés sur feuille libre en leur donnant une forme proche de la version éditée avec illustration en couleurs et texte en noir (au feutre-pinceau, selon ses habitudes), où l'on peut voir également différents états du texte et de l'image.

Pour le volet «Liberté », l'analyse des carnets nous intéresse particulièrement, en raison de la place qu'ils occupent et du rôle qu'ils jouent dans le processus créatif de l'auteure. Ils viennent prendre la suite, ici, d'un premier travail de recherche et d'exploration du texte et de l'illustration sur feuille libre, et correspondent à ce titre à un pré-projet d'édition. Le passage par le carnet permet, en effet, à l'auteure de donner à son œuvre une forme qui correspond au format de l'édition : le carnet devient album, avec la tourne des pages et la matérialité de la pliure qui viennent l'une et l'autre perturber la continuité entre les pages. L'illustration y trouve son expression quasi définitive (traits et couleurs). Cependant, malgré cette forme qui le rapproche de la version éditée, le carnet n'en demeure pas moins le lieu de modifications, d'interrogations, comme si le travail de réécriture pouvait être sans fin. Et c'est précisément ce retour incessant sur le texte que nous souhaiterions

4. Nous appelons «version éditée» l'état du texte et de l'image retenu pour l'édition.

5. Le fonds Rosenstiehl est entré à l'IMEC en 2007. Il contient 61 boîtes d'archives ; 60 imprimés datés de 1965 à 2009, catalogués sous la cote 378RSN/1 - 378RSN/61.

6. Nous appelons «version» «chacun des états du texte comportant des variantes ou des modifications du fait de l'auteur» (www.cnrtl.fr). 


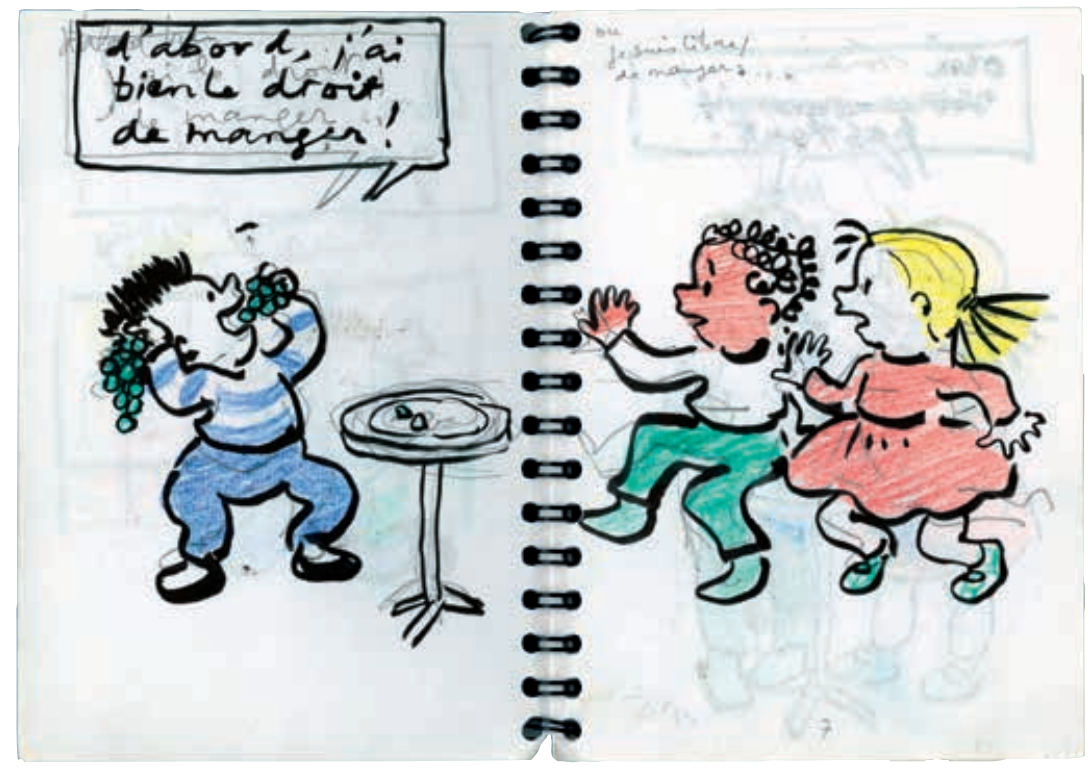

Fig. 2 : Première double page du carnet bleu à spirales $(10,5 \mathrm{~cm}$ x 14,8 cm), numérotée 6-7. Croquis pour «Liberté». Cote : 378RSN/22/0. Titre du dossier : Croquis pour Mimi Cracra.

(C) Archives Agnès Rosenstiehl/IMEC.

mettre au jour à travers l'analyse de quelques pages extraites d'un petit carnet bleu à spirales, avec papier dessin blanc $(10,5 \times 14,8)$, contenant des traces d'élaboration (note de régie) et de modification.

Le carnet s'ouvre sur le volet «Liberté», avec numérotation de chaque page au crayon (centre bas), à partir de 3 (conformément à la numérotation des albums, hors pages de garde), avec une configuration qui annonce celle de l'album publié : la double page du carnet correspond à la double page de l'album avec une répartition majoritaire texte/image à gauche et image seule à droite ou l'inverse ${ }^{7}$ (comme décrit supra).

Sur la première double page numérotée 6-7, reproduite ici (fig. 2), le personnage principal (illustration en page de gauche) vient se servir des raisins qu'il trouve sur la table, sans se soucier de ceux à qui ils appartiennent et qu'il est en train de spolier. Le texte ( «D'abord, j' ai bien le droit de manger ! ») est écrit en haut à gauche dans un phylactère au crayon à papier d'abord, puis au feutre-pinceau noir ensuite, sans que les deux ne se superposent, l'auteure laissant ainsi les deux états coexister. Sur la page de droite, on peut relever une note de régie au crayon à papier contenant une variante possible du texte, manifestant ainsi l'hésitation de l'auteure (hésitation pleinement exprimée par la conjonction de coordination «ou», qui situe précisément l'alternative sur le plan «métascriptural») : «ou je suis libre de manger!» La version éditée retiendra la seconde qui pose la liberté plutôt que le droit: «D'abord, je suis libre de manger!» L'illustration est réalisée au feutre-pinceau noir pour les contours et en couleurs avec traces du crayon à papier initial (esquisse) : page de gauche, notre personnage principal; page de droite, la réaction de surprise des protagonistes.
Sur la double page suivante numérotée 8-9 reproduite ici (fig. 3), plusieurs niveaux de texte apparaissent :

- un premier état du texte écrit au stylo à bille bleu : «nous aussi ! », encadré d'un phylactère ;

- le texte a été barré avec le même stylo et remplacé par «Pas tout!», l'auteure mettant ainsi en avant non plus l'opposition de deux libertés - celle de notre personnage principal confrontée à celle des deux autres personnages, mais plutôt l'idée de partage et de fraternité : mon désir peut être compatible avec celui des autres, à condition que j'aie ce souci de l'autre;

- le texte (avec le phylactère) est repris au feutre-pinceau noir (comme vu précédemment) : les deux états ne se superposent pas complètement, c'est ce qui rend possible l'identification de la chronologie dans l'écriture du texte. L'auteure modifie de nouveau son texte avec un nouvel essai réalisé directement au feutre-pinceau noir : «oui/nous aussi », faisant réapparaître ainsi le texte auparavant biffé et rejeté au profit de «pas tout !». Elle revient à son choix initial en barrant de nouveau, mais cette fois au feutre, «nous aussi », avant de passer au pinceau-feutre noir les contours de «pas du tout», obtenant ainsi le texte «oui [...] pas tout !», auquel elle va ajouter un «mais» suivi de points de suspension après «oui », un peu plus tard, comme en témoigne le changement d'épaisseur du feutre.

7. La répartition texte/image se fait comme suit : texte/image à gauche et image seule à droite pour les doubles pages 2-9, 12-13, 16-17, 21-22, 24-25 et l'inverse pour les doubles pages 10-11, 14-15, 18-19, 22-23, 26-27; pour les deux dernières doubles pages, le texte est écrit en haut de page de part et d'autre de la pliure. 


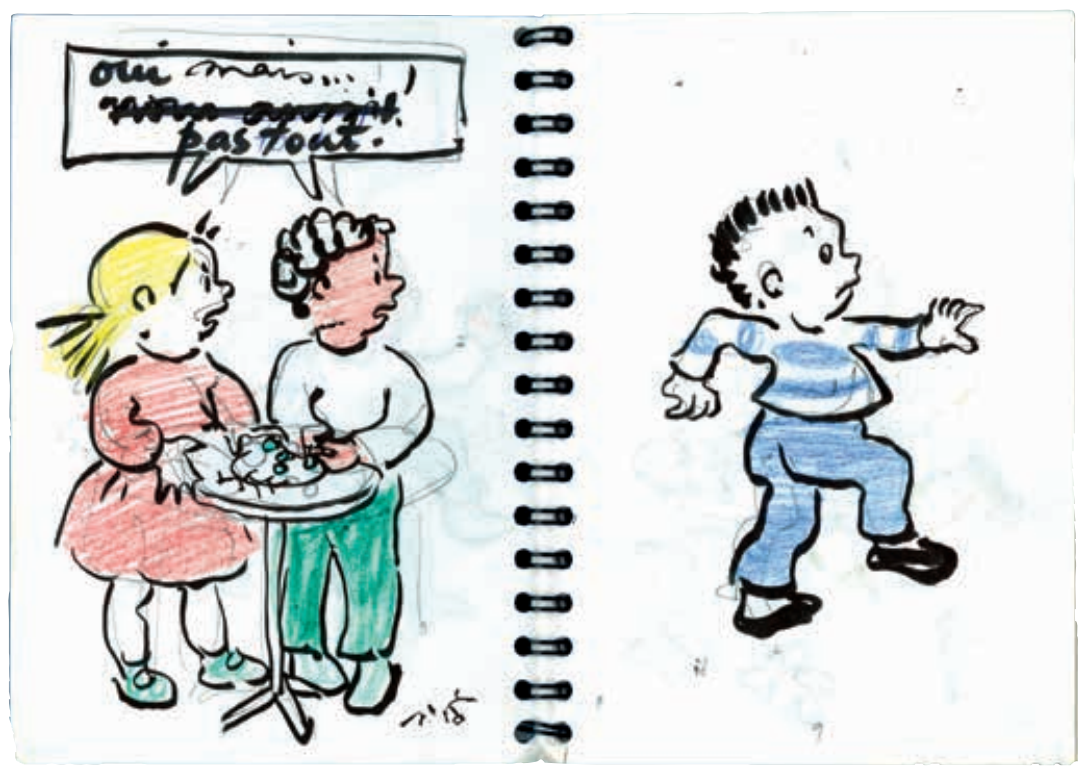

Fig. 3 : Deuxième double page du carnet bleu à spirales $(10,5 \mathrm{~cm} \times 14,8 \mathrm{~cm})$, numérotée 8 -9. Croquis pour «Liberté» Cote : 378RSN/22/0. Titre du dossier : Croquis pour Mimi Cracra.

(c) Archives Agnès Rosenstiehl/IMEC.

Le texte ainsi modifié correspond à la version éditée : «Oui mais... pas tout!» qui insiste sur le partage au lieu de renvoyer un égoïsme à un autre.

Ce focus sur ces quelques pages de l'un des carnets de l'auteure nous renseigne sur l'une de ses modalités d'écriture, par essais successifs, où elle revient à des essais antérieurs pour les mettre de nouveau à l'épreuve avant de les abandonner et de revenir aux choix opérés en dernier, une écriture par retour sur le même, qui lui permet d'avancer et auquel l'illustration vient se mêler. Afin de poursuivre l'analyse du processus créatif de l'auteure, nous souhaiterions porter notre attention sur quatre autres documents préparatoires de «Liberté» avec lesquels ces quelques pages de carnets vont pouvoir entrer en résonance.

\section{«Liberté» : un parcours à l'œuvre}

C'est la nature même des quatre documents retenus pour l'analyse de ce premier volet qui va nous permettre d'appréhender le processus créatif de l'auteure :

- un canevas comprenant les thèmes envisagés par double page en une page $\mathrm{A} 4$ format paysage répartie en 24 cases correspondant chacune à une double page d'un format album (cf. infra fig. 4);

- trois états successifs d'un même chemin de fer en une page A4 format paysage répartie en 16 cases (et non plus en 24). Ces cases matérialisent les 16 doubles pages du format album qui sera retenu pour l'édition (soit 32 pages pour ce volet «Liberté»). Le premier document, dans la chronologie, désigné par la suite «premier état du chemin de fer», est réalisé au crayon à papier (cf. infra fig. 5); le deuxième (désigné par la suite «deuxième état du chemin de fer») est la reprise au net de ce premier document avec le texte écrit au feutre (cf. infra fig. 6), le troisième (désigné par la suite «dernier état du chemin de fer», avant le passage au carnet) est une version modifiée au crayon à papier à partir du deuxième document, visiblement photocopié (cf. infra fig. 7). Le même type de documents préparatoires se retrouve pour chacun des trois volets. Ce travail d'élaboration successive du texte et de l'image, que nous allons analyser, trouve un prolongement dans les carnets qui sont le lieu de développement du travail sur l'illustration (colorisation et aboutissement du trait) et d'un énième retour sur le texte; mais aussi ses prémices, les carnets contenant des espaces de recherche, où l'auteure envisage en une page (ou deux) les grands axes définitoires de la liberté (la liberté de mouvement, le droit d'avoir un nom, de vivre, d'aller à l'école) qui seront repris dans le texte. Ce même processus de recherche se retrouve à propos du volet «Fraternité» dans l'un des carnets mentionnés avec des pistes de questions à traiter et les problèmes que pose la fraternité («Bâtir ensemble? [cabane, château]; Voir un frère en l'Autre?; Partager, redistribuer [gâteau] richesses?; Reconnaissance fratrie [identité] ?; Famille humaine [ $\neq$ animale]»).

\section{Le canevas de l'œuvre ou De la recherche des idées (fig. 4)}

L'auteure envisage, à cette étape du processus créatif, un plan en trois parties :

Une première, faisant office de préambule, qui pose le cadre institutionnel de la liberté en France en trois doubles pages (avec une «histoire de la liberté» et sa place dans la 


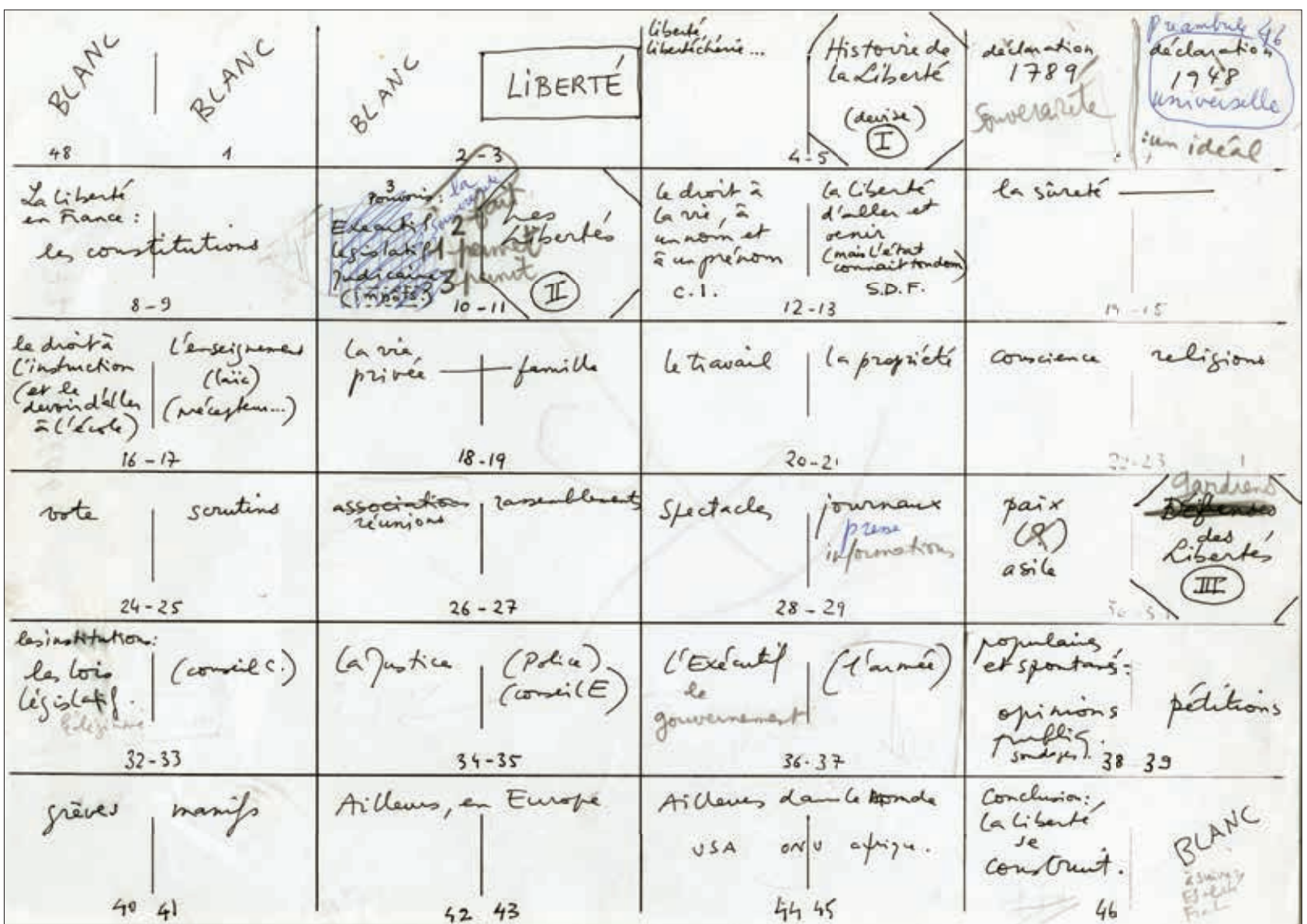

Fig. 4 : Canevas «Liberté»-Format : 29,7 cm × $21 \mathrm{~cm}$. Cote : 378RSN/22/0. Titre du dossier : Croquis pour Mimi Cracra.

(๑) Archives Agnès Rosenstiehl/IMEC.

devise républicaine; la Déclaration des droits de l'Homme et du citoyen de 1789, la Déclaration universelle des Droits de l'Homme de 1948, les constitutions et les trois pouvoirs - législatif, exécutif et judiciaire - comme garants des libertés).

Une deuxième partie développant les différentes libertés auxquelles peuvent prétendre les citoyens (dix doubles pages) et qui reposent sur les droits fondamentaux :

- ceux des individus d'abord, avec le «droit à la vie», à une identité, «un nom et un prénom» (12); le droit et la liberté d'aller et venir (13); le droit à la sûreté (14-15); le droit à la vie privée et à l'intimité (18-19); le droit à la propriété et la liberté d'entreprendre («travail», 20-21); la liberté de culte et d'opinion (22-23; 24-25);

- les droits et les libertés collectives qui concernent chaque individu au sein de la collectivité : la liberté de réunion et d'association (26-27); la liberté de la presse (29);

- les droits sociaux garantis par l'État : le droit à l'instruction (16-17) et à la culture («spectacles», 28).
Une troisième partie présentant les «gardiens des libertés » venant remplacer les «défenses des libertés » (sept doubles pages) : les institutions (la loi, le Conseil constitutionnel; la Justice et la police; l'Exécutif et l'armée); à l'échelle du peuple ( «gardiens populaires et spontanés»: sondage/opinion publique, les pétitions, les grèves/manifestations) avec une comparaison avec l'étranger («Ailleurs en Europe», «Ailleurs dans le monde»).

Une conclusion : «la liberté se construit».

Si l'auteure opte ici pour une approche législative (juridique) et politique (démocratique) de la liberté, elle va y renoncer par la suite pour la ramener à une expérience des manifestations de la liberté : comment s'exerce notre liberté? Quelles en sont les conditions? Les limites de la liberté sont celles qu'imposent au sujet le respect de la liberté d'autrui et le respect de la loi. Par ailleurs, l'auteure adopte le point de vue de l'enfant qui découvre ce qu'est la liberté et en expérimente les conditions dans la confrontation de ses désirs à ceux des autres. Il découvre dans le rapport 


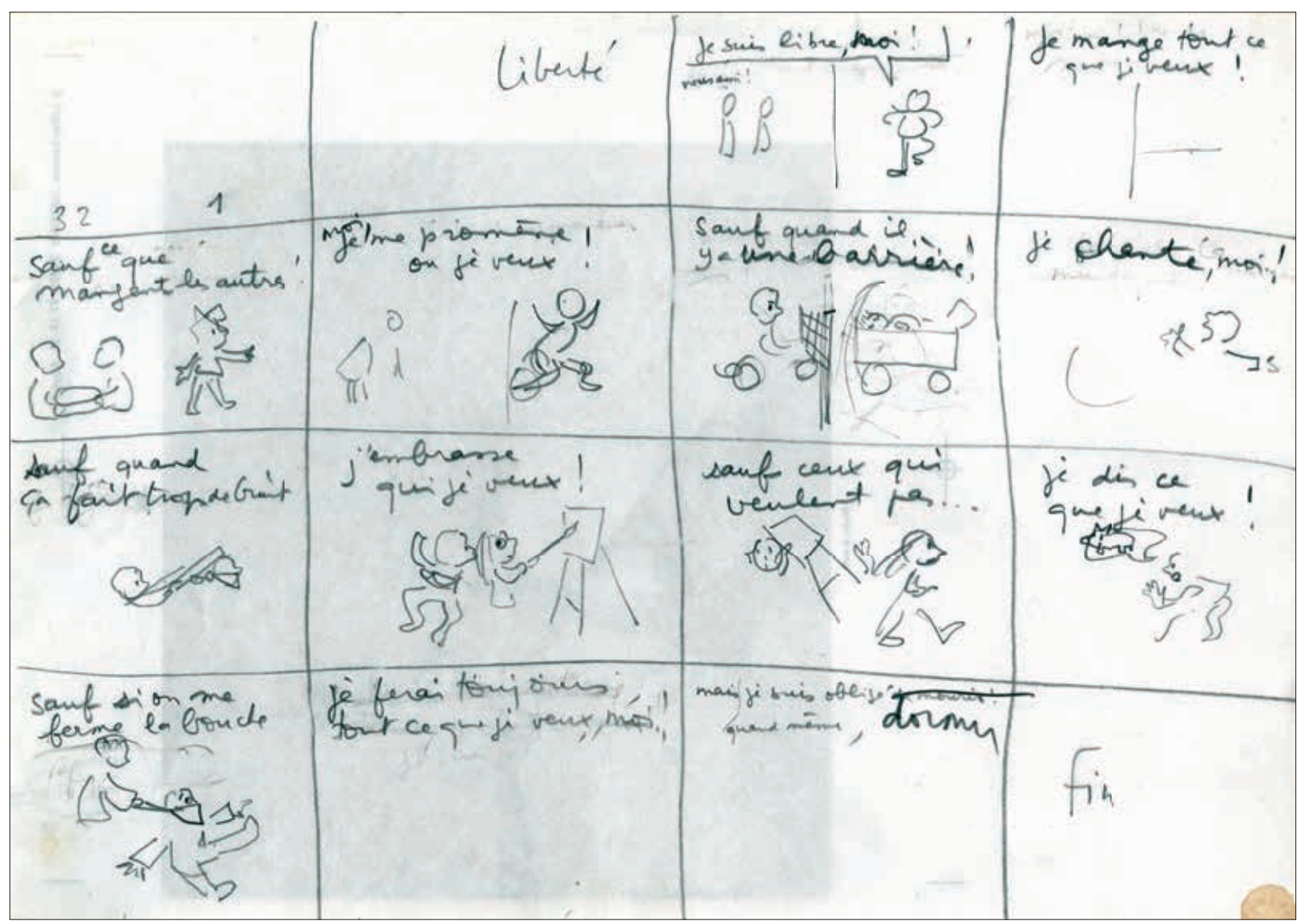

Fig. 5 : Premier état du chemin de fer «Liberté »-Format : 29,7 cm $\times 21 \mathrm{~cm}$. Cote : 378RSN/22/0. Titre du dossier : Croquis pour Mimi Cracra. (C) Archives Agnès Rosenstiehl/IMEC.

à l'Autre le cadre d'exercice de la liberté qui en assure aussi la protection.

\section{De la constitution du chemin de fer}

Il s'agit là d'un processus en plusieurs étapes réalisé à partir du canevas que nous venons d'analyser. Nous avons pour le corpus qui nous intéresse deux états successifs dans l'élaboration du chemin de fer de ce que sera l'album édité; le premier document - premier état du chemin de fer - est aussi le premier état de la planche.

\section{- Premier état du chemin de fer : une esquisse (fig. 5)}

Dans ce document, le texte est au crayon à papier et l'illustration à l'état de première esquisse sur la double page. L'auteure épure son texte initial de tout ce qui est abstrait pour l'ancrer dans une expérience individuelle de la liberté et des conditions de son existence. Par une simplification maximale, elle tente d'amener le jeune lecteur/auditeur à réfléchir par lui-même contre tout discours informatif ou moralisateur.

Le personnage principal (un jeune enfant) revendique sa liberté («je suis libre, moi! » [4-5] avec ajout en page 4 de «nous aussi », revendication faite par ceux qui entourent le narrateur-personnage; ce dernier énonce ensuite un champ possible de déploiement, en fait l'expérience avant de poser lui-même les limites à sa liberté (introduites par «sauf...») : «je mange tout ce que je veux» (6-7)... « sauf ce que mangent les autres » $(8-9)$; « moi je me promène où je veux... sauf quand il y a une barrière»; on retrouve ici la mention de la liberté de circulation (page 13 du canevas, cf. supra fig. 4). Sont ensuite revendiqués les droit de chanter (14-17), d'embrasser qui l'on veut (18-21), de dire ce que l'on veut (22-25).

L'auteure, en dernière page, remplace «mourir» par «dormir» opposant une fin tragique (vanité du monde) à une fin plus légère qui fait sourire le lecteur : «Je ferai 


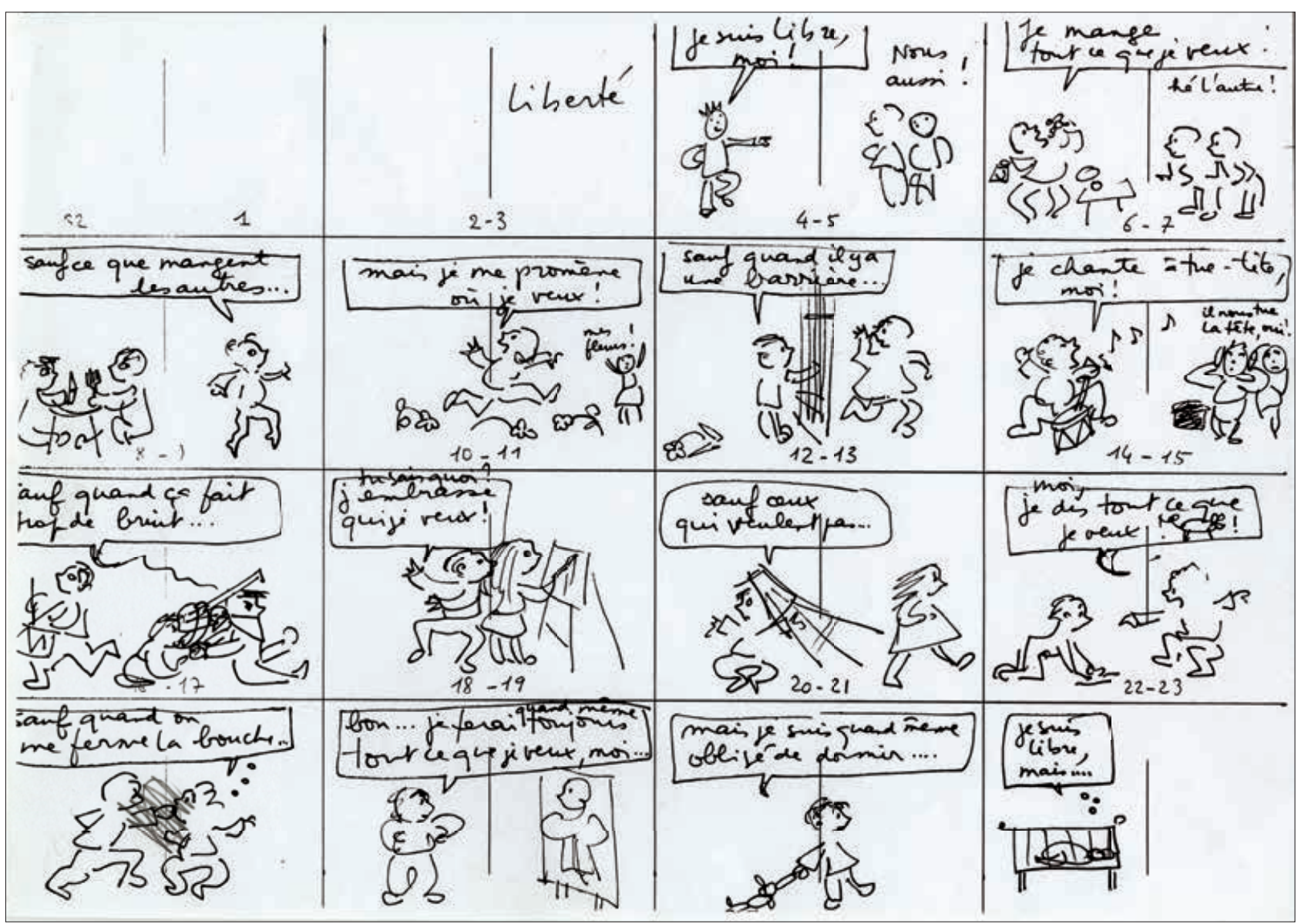

Fig. 6 : Deuxième état du chemin de fer «Liberté»-Format : $29,7 \mathrm{~cm} \times 21 \mathrm{~cm}$. Cote : 378RSN/22/0. Titre du dossier : Croquis pour Mimi Cracra.

(c) Archives Agnès Rosenstiehl/IMEC.

toujours tout ce que je veux, moi !... Mais je suis obligée de dormir quand même». Si la mort était la barrière naturelle à la liberté, le sommeil devient ce besoin vital contre lequel l'enfant ne peut pas lutter, quand bien même son désir le pousserait à vouloir se soustraire à cette nécessité.

\section{- Deuxième état du chemin de fer (fig. 6)}

Cette version correspond à une stabilisation du texte et de l'image : le texte est écrit au feutre noir et intégré dans des phylactères de forme rectangulaire et le geste dans l'illustration se précise ainsi que la répartition sur les deux pages de l'illustration qui se systématise avec à gauche ce que fait le personnage en quête de liberté et à droite la réaction de ceux qui l'entourent. Quelques légères modifications de surface ont été apportées au texte :

- pages 10-11, «moi, je me promène où je veux » devient «mais je me promène où je veux »;
- pages 14-15, «je chante, moi !» est complété par «à tue-tête» («je chante à tue-tête, moi !») et l'illustration répartie sur les deux pages, avec à gauche, notre chanteur à tue-tête et à droite la réaction de son entourage avec ajout de texte «il nous tue la tête, oui !»;

- pages 18-19, une adresse au lecteur, «tu sais quoi ?», est ajoutée au texte, avant «j'embrasse qui je veux!» ce qui rend aussi à l'acte du personnage (le baiser volé) une désinvolture manifeste, tout en résonnant comme un langage enfantin;

- pages 24-25, l'illustration a été modifiée : le bâillon mis à celui qui parle en réaction à ses propos probablement blessants ou dérangeants a été remplacé par un coup porté à la bouche, à la manière d'une estocade, puis biffé après coup au crayon à papier (probablement au moment de la révision de ce second état du chemin de fer, réalisée à partir d'une (photo)copie de ce dernier - cf. fig. 7); 


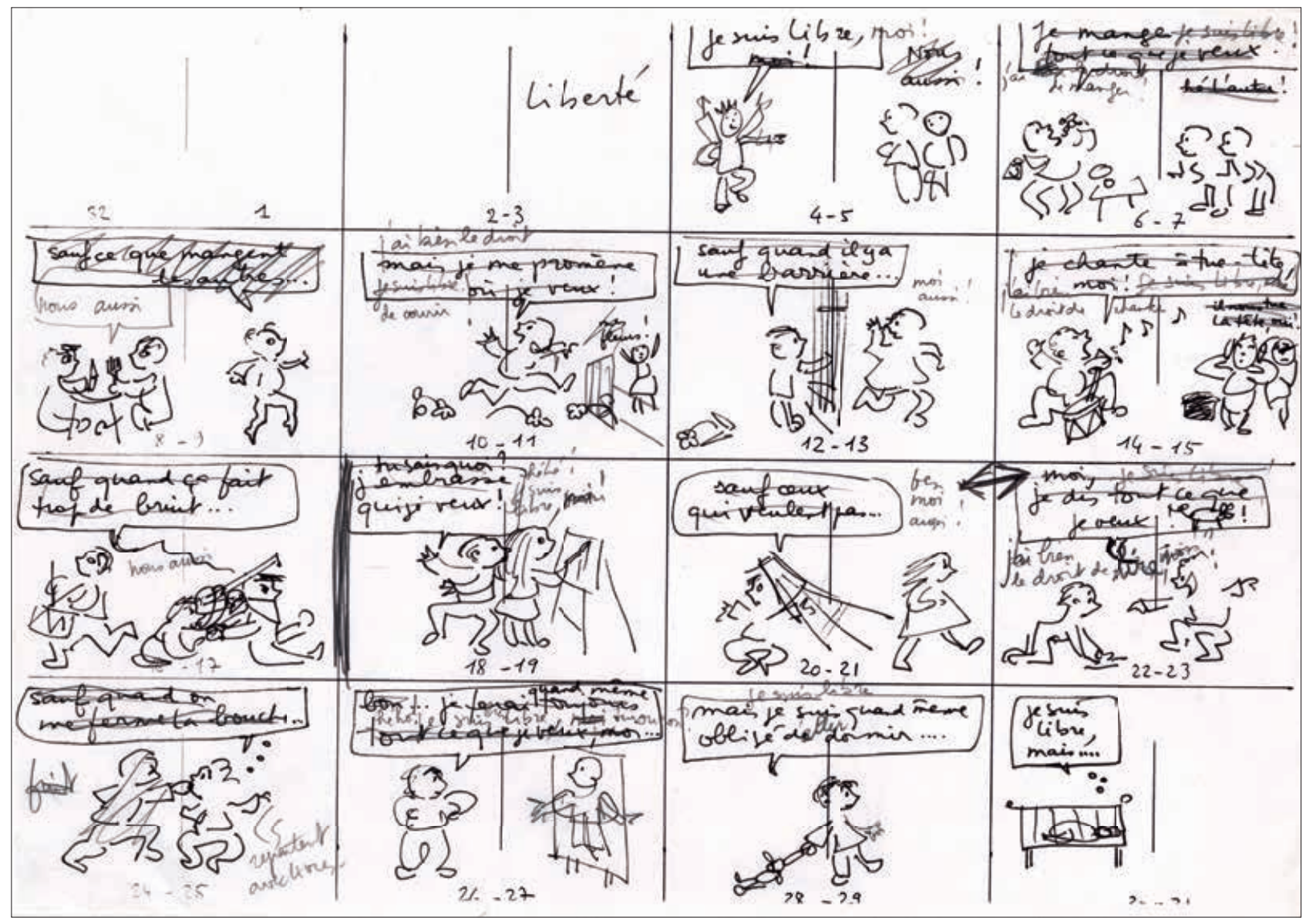

Fig. 7 : Dernier état du chemin de fer «Liberté», modifié au crayon à papier - Format : 29,7 cm $\times 21 \mathrm{~cm}$. Cote : 378RSN/22/0. Titre du dossier : Croquis pour Mimi Cracra.

(c) Archives Agnès Rosenstiehl/IMEC.

- enfin, une page a été ajoutée (page 30) venant prolonger la précédente double page : le sommeil comme frein naturel à la liberté, mais temporaire et ô combien nécessaire.

\section{Dernier état du chemin de fer ou De la révision du texte (fig. 7)}

Ce troisième état du chemin de fer, qui est une reprise de la version précédente (fig. 6) avec modifications principalement sur le texte (à l'exception des illustrations p. 4 et 27), laisse entrevoir le travail de révision, de retour sur le texte. La principale transformation se fait dans la formulation de la liberté par le recours aux droits : «j'ai le droit de...», qui fait émerger l'idée que mes droits sont aussi des devoirs envers les autres («ma liberté s'achève où celle de l'autre commence »). C'est dans cette version que se met en place le principe de fonctionnement de l'album décrit plus haut à propos de la présentation de chacun des volets constitutifs de l'œuvre : les actes du personnage principal ont une incidence sur son entourage qui y réagit, les doubles pages se lisant alors par deux, de manière séquentielle. Certaines réactions présentes dans le texte ont été retirées au seul profit de l'illustration (pages 4-5 : «Nous aussi»; pages 6-7 : «hé 1'autre!»; pages 14-15 «il nous tue la tête, oui!»); et d'autres ajoutées (pages 8-9 et 16-17 «Nous aussi»; pages 12-13 «Moi aussi !»; pages 20-21 «ben moi aussi !»).

Les limites imposées à la liberté du personnage principal ne le sont plus dans une structure syntaxique introduite par «sauf» (pages 6-7 : «Je mange tout ce que je veux... sauf ce que mangent les autres »). C'est un véritable dialogue qui se met en place : l'enfant sort du dialogue de soi à soi (il ne s'adresse plus à lui-même) pour se confronter à la réaction des autres, la liberté avancée trouvant ainsi chez l'autre une objection, celle de sa propre liberté. Il semblerait que la liberté d'un individu ne puisse exister qu'à la 
condition que ce même individu accepte de prendre en compte l'existence de l'Autre selon le principe de fraternité, qui consiste à voir cet Autre comme un frère, comme un autre soi-même, pensant et désirant. Cette liberté revendiquée s'énonce alors en termes de droit : «J'ai le droit de manger !... Nous aussi!» (pages 6-8); «J'ai bien le droit de courir» (pages 10-11), qui remplace «mais, je me promène où je veux », barré et remplacé par «je suis libre de courir»; réponse en pages 12-13 «Moi aussi!». Cette même hésitation entre «je suis libre de...» et «j' ai le droit de...» se retrouve en pages :

- 14-15: «Je chante à tue-tête, moi» $\rightarrow$ « Je suis libre de chanter» $\rightarrow$ «J'ai bien le droit de chanter»;

- 18-19: «Tu sais quoi? J'embrasse qui je veux!» $\rightarrow$ «Héhé! Je suis libre, moi!»;

- 22-23: «Moi, je dis tout ce que je veux» $\rightarrow$ «Je suis libre! » $\rightarrow$ « J'ai bien le droit de dire, non? » où « dire» est remplacé par «lire» (en surimpression), texte retenu pour l'édition. La double page suivante 24-25 contient des éléments de modification : le coup porté est rayé et une indication pour le changement de l'illustration a été ajoutée : «repartent avec livres»;

- 26-27: «Bon, je ferai toujours quand même tout ce que je veux, moi...» $\rightarrow$ « hé ho, je suis bien libre, moi oui ou non?»;

- 28-29: «mais je suis quand même obligé de dormir d'aller dormir...» // «je suis libre».

\section{De la place de l'illustration dans le processus créatif}

$\mathrm{Si}$, dans ce corpus, l'illustration semble émerger une fois le canevas de l'œuvre posé dans ses grandes lignes, les versions suivantes, qui sont une exploration et un développement de ce premier état de l'œuvre, témoignent d'une co-élaboration du texte et de l'image : au fur et à mesure que le texte s'affine, l'illustration prend de l'épaisseur, sans qu'il nous soit possible d'affirmer qui du texte ou de l'illustration agit sur l'autre en premier. C'est dans les carnets, qui se rapprochent par leur format de la version éditée, que l'illustration trouve une forme aboutie, où apparaissent les contours en noir et les couleurs. Et pourtant, comme nous l'avons vu précédemment à propos d'un extrait du carnet bleu relatif à ce volet «Liberté», l'élaboration fine de l'illustration ne fige pas pour autant le texte.
Il semblerait cependant qu' un retour incessant sur le texte entraîne une relecture de l'image. Nous souhaiterions revenir ici sur les pages 22-25 des deux derniers états du chemin de fer (fig. 6 et 7) où «Moi, je dis tout ce que je veux» (texte du deuxième état du chemin de fer, fig. 6) est successivement modifié en «Je suis libre!», puis «J'ai bien le droit de dire, non? » où enfin «dire» est remplacé par «lire». La modification du texte a une incidence sur l'illustration, car l'illustration fait résonner le texte d'une certaine façon : ici, la violence de la parole (dire tout ce que l'on veut, y compris ce qui blesse et attaque) entraîne une réponse tout aussi violente, manifestée dans l'illustration par le bâillon, puis par le coup au visage. Le choix final de la lecture (au lieu de la parole) induit un tout autre rapport à l'autre : non plus un rapport conflictuel où une liberté porte atteinte à celle d'un autre, mais un rapport d'émulation; émulation par laquelle le comportement de l'un donne envie à l'autre d'en faire autant pour y trouver le même plaisir/bénéfice.

Si l'exploration de documents relatifs à la genèse de ce premier volet «Liberté» nous a permis d'approcher le processus créatif de l'auteure, nous souhaiterions à présent porter notre attention sur le deuxième volet de l'œuvre, «Égalité». Il rend compte, en effet, d'un type de parcours d'écriture avec revirement (qui se manifeste ici par une transformation assez radicale du texte initial) où l'attention portée au lecteur va venir modifier la forme du texte afin qu'il réponde au projet visé par l'auteure : faire réfléchir le public auquel elle s'adresse sur des questions essentielles, ici celle de l'égalité de tous devant les droits - principe fondamental - en dépit des différences qui nous caractérisent (qu'elles soient liées à notre physique, à notre sexe, à notre statut social, à nos convictions, etc.).

\section{«Égalité»}

Ce deuxième volet nous confronte à un problème intéressant : sont conservées dans les archives plusieurs versions, selon le processus de création mis au jour jusque-là (canevas, plusieurs états successifs d'un chemin de fer, carnets avec différents états du texte et de l'illustration) d'un même texte qui ne sera pas repris pour la version éditée. Dans le même temps, il est possible d'observer des avant-textes comportant un profond remaniement du 


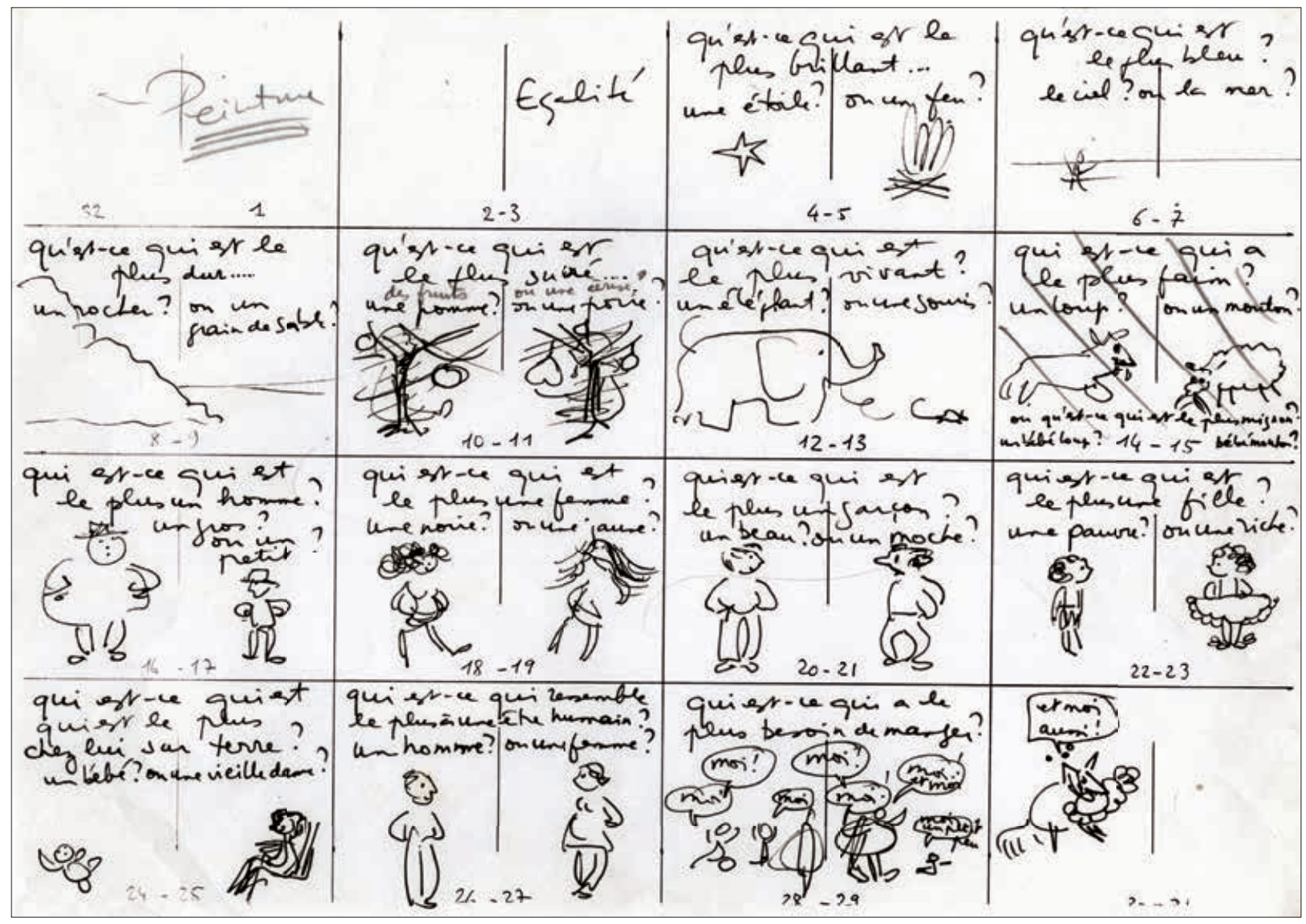

Fig. 8 : Canevas «Égalité»-Format : $29,7 \mathrm{~cm} \times 21 \mathrm{~cm}$. Cote : 378RSN/22/0. Titre du dossier : Croquis pour Mimi Cracra.

(c) Archives Agnès Rosenstiehl/IMEC.

texte initial, dans deux versions déjà élaborées (l'une dans l'un des carnets mentionnés ci-dessus et l'autre à travers quelques montages texte/illustrations en vue de constituer la maquette pour l'édition) qui vont aboutir à la version éditée. La comparaison de ces deux états du texte (le texte initial $v s$ le texte profondément remanié) va nous permettre, si ce n'est de mettre au jour, du moins de faire des hypothèses sur les motivations des changements opérés et sur ce qu'elles nous apprennent des considérations d'un auteur qui écrit pour la jeunesse. Pour ce que nous avons appelé le texte initial, nous analyserons un chemin de fer au format A4 paysage, réparti sur 16 cases $^{8}$, avec texte écrit au stylo noir et comportant des modifications au crayon à papier : comme nous l'avons observé à propos du dernier état du chemin de fer du volet «Liberté» (cf. fig. 7), ce document est vraisemblablement une (photo)copie de la planche immédiatement antérieure à partir de laquelle l'auteure procède à des modifications manuscrites. Nous mentionnerons également un second état du texte (texte initial profondément remanié), proche de la version éditée, contenu dans l'un des carnets relatifs à l'œuvre (carnet vert mentionné ci-dessus).

Le texte initial du volet «Égalité» (fig. 8) est construit sur un système de comparaison entre deux réalités au sein d'une interrogation qui invite le lecteur à choisir l'une plutôt que l'autre : «Qu'est-ce qui est le plus... ? X ou Y» ou «Qui est-ce qui... le plus? X ou Y». La présence du superlatif relatif induit un positionnement sur une échelle de valeurs entre les deux entités comparées et le singulier contenu dans la question incite le lecteur à ne choisir qu'un seul des deux éléments mis en balance. Or, l'idée sous-jacente de l'auteure, c'est d'amener le jeune lecteur à conclure de ces oppositions que finalement, quoi que l'on soit ou

8. Nous rappelons que ces 16 cases matérialisent, comme c'était le cas pour le volet «Liberté», les 16 doubles pages du format album qui sera retenu pour l'édition (soit 32 pages pour ce volet «Égalité ») 
qui que l'on soit, derrière les différences, nous avons les mêmes droits. La conjonction de coordination «ou» a deux sens possibles en français ${ }^{9}$ : soit elle marque la disjonction exclusive (l'un ou l'autre), soit la disjonction inclusive (l'un ou l'autre ou les deux), elle ne permet pas de refuser l'alternative (ni l'un ni l'autre). Derrière cette structure syntaxique de disjonction, ce qu'attend l'auteure de son lecteur, c'est non pas qu'il retienne l'opposition (que soutient un «ou » disjonctif), mais plutôt qu'il fasse une lecture inclusive de la conjonction («les deux»), voire qu'il refuse l'alternative et la comparaison qu'elle contient («ni l'un ni l'autre»).

C'est en provoquant des oppositions que l'auteure tente de faire remarquer au lecteur qu'elles ne sont pas tenables et qu'elles n'ont pas de sens au nom de l'unité et de l'égalité. L'auteure tente de rendre accessible et explicite (?) au jeune lecteur la notion d'égalité de droit derrière les différences de couleur de peau, de sexe, de vivant (entre les règnes). Mais le jeune lecteur, confronté depuis le début à ce principe (qui fonctionne jusqu'à la double page 14-15 où l'un est plus... que l'autre), ne parvient pas à en sortir et se retrouve devant une aporie quand on change de registre : comment choisir qui est le plus humain entre un garçon et une fille ou entre une Blanche et une Noire? Les enfants, alors habitués jusque-là à un type de raisonnement par comparaison, peuvent être tentés de le poursuivre et de proposer que la Blanche est plus humaine que la Noire, réponse venant ruiner le principe même de l'album qui consiste à poser l'égalité en droits indépendamment des différences de chacun.

Dès lors, la version éditée du texte (cf. ci-dessous) tiendra compte de ce risque de lecture afin de ne pas provoquer chez le jeune lecteur une lecture aux antipodes de l'éveil des consciences que vise l'œuvre.

Texte intégral de la version éditée :

Tous les bébés qui naissent font partie de la famille des êtres humains.

On peut naître couleur du blé, couleur du soir,

Couleur du soleil, couleur de la rose,

Couleur du chocolat, couleur du café,

Couleur du caramel, couleur du pain,

Couleur jus de pêche ou jus d'abricot,

Couleur de l'encre ou couleur du papier,

Mais dans la République française, tout le monde a les mêmes droits : par exemple, tout le monde a le droit de dormir à l'abri, celui-ci et celui-là...

tout le monde a le droit d'aller à l'école, celui-ci et celui-là... tout le monde a le droit de penser, celle-ci et celle-là... tout le monde a le droit au respect, celle-ci et celle-là... et tout le monde a le droit de manger!

— Hé hé ! Moi aussi, j'ai envie de manger. — Hé ! C'est pas juste!

Voici une étude comparée de ce texte initial (fig. 8) et de la version éditée (reproduite ci-dessus) :

Contrairement au premier état du texte (texte initial), l'auteure s'est focalisée ici sur l'humain et l'égalité des droits malgré les différences d'âge, de sexe, de classe sociale, de culture (religion), le handicap, en supprimant tout ce qui relevait de la nature (étoile/feu; ciel/ mer; rocher/sable; pomme/poire; éléphant/souris; loup/ mouton). En effet, dans le texte initial, les six premières doubles pages mettent en balance deux entités en fonction d'une particularité : la brillance, la couleur, la dureté, le caractère sucré, puis survient une première rupture en pages 12-13 : «qu'est-ce qui est le plus vivant?». Dans l'énoncé de cette question, se lit une rupture sémantique avec « vivant» puisque l'auteure introduit ici une variation en degré sur un adjectif qui n'est en principe pas gradable (contrairement à «brillant», «bleu», «dur»). Si l'auteure s'autorise cette licence, c'est par un jeu d'association, de brouillage volontaire, de l'ordre du monde avec celui de la langue, où l'éléphant serait «plus vivant que » la souris, selon un ordre de grandeur reposant sur une comparaison de la corpulence des deux animaux retenus. Par ailleurs, ce jeu d'association (fondée sur une relation de taille, mais aussi de couleur) est redoublé par une proximité culturellement fantasmatique entre les deux animaux, où la souris pourrait en s'introduisant dans la trompe de l'éléphant, rendre fou ce dernier. Ainsi le pachyderme aurait-il à craindre d'un animal plus petit que lui, comme en témoigne l'univers des fables.

C'est d'ailleurs à ce même univers de référence qu'appartient le couple de la seconde rupture, loup-mouton, qui se produit en pages 14-15, avec deux versions concurrentes du texte : «Qui est-ce qui a le plus faim? Un loup ou un

9. M. Arrivé, F. Gadet, M. Galmiche, La Grammaire d'aujourd'hui, Paris, Flammarion, 1986, p. 196-197. 
mouton?» et «Qu'est-ce qui est le plus mignon? un bébé loup? un bébé mouton?». Ici, le rapprochement loup/ mouton pourrait trouver sa justification dans la représentation symbolique des deux animaux : dans l'univers des fables où les animaux sont anthropomorphisés, le loup incarne le monde sauvage par opposition au mouton qui, lui, incarne le monde domestiqué dans lequel il est aussi l'emblème de la soumission (comme renoncement à l'exercice de son intelligence). L'opposition des deux animaux sur le critère de la faim (prédicat gradable ici) présuppose la convocation par le lecteur de l'expression métaphorique «avoir une faim de loup ». Cette première tentative sera abandonnée au profit d'une seconde comparaison reposant sur une appréciation de type affectif («qu' est-ce qui est le plus mignon?») et une mise en balance non plus des deux animaux, mais d'une catégorie du vivant qui rend cette opposition caduque : «bébé ». Dès lors, l'auteure invite son lecteur à raisonner ainsi : puisque par principe tous les «bébés » sont «mignons», à la question «qui du loup ou du mouton est le plus mignon?», on attend «les deux» comme réponse.

À la suite de ces deux ruptures, l'opposition change de registre pour ne reposer que sur des caractéristiques humaines : qu'est-ce qui fait l'homme? son poids; la femme ? sa couleur de peau; le garçon? sa beauté/son physique; la fille? sa fortune; qu' est-ce qui justifie sa place sur terre? le fait d'être jeune ou vieux / l'âge; quel est le signe de notre appartenance au genre humain? le sexe; à qui s'applique le besoin de manger le plus grand ? C'est en fait un besoin vital qui nous rassemble tous.

La catégorisation des questions que pose Agnès Rosenstiehl nous informe sur la mise en œuvre de son processus créatif et nous fait mesurer tous les sauts qualitatifs auxquels elle s'adonne avant de se centrer sur l'humain : elle laisse libre cours à son inventivité ; ce qui vient lui faire abandonner des possibles, c'est ce qui peut faire exploser son projet (faire comprendre l'un des trois volets de la devise républicaine à des enfants de 3-6 ans). En effet, parler d'égalité entre un éléphant et une souris, ou une étoile et un feu ne peut pas déclencher chez le jeune lecteur une prise de conscience de ce qu'est l'égalité (de droits); si l'opposition loup/mouton nous dit quelque chose de l'homme, par la tradition littéraire qui les a anthropomorphisés, l'auteure finit pourtant par l'abandonner quand son second essai annule l'opposition loup/mouton au seul profit de la catégorie «bébés » et du jugement esthétique et affectif qu'il engendre nécessairement («mignon»). Si le projet préexiste à l'écriture, la forme, quant à elle, se construit dans et par l'écriture, dans un jeu d'explorations multiples, qui à un moment donné de son avancement intègre le souci du lecteur.

C'est à partir d'un tel cheminement que la première aporie signalée plus haut, qui incite le jeune lecteur à préférer la femme blanche à la femme noire quand il s'agit de déterminer «ce qui est le plus une femme», peut alors être levée. Pour y parvenir, l'auteure décline dans la version éditée la différence de couleur de peau, en conservant certes l'opposition noir/blanc, mais en la déployant et en passant en revue tout un panel de couleurs de peau qui rend l'humain singulier, l'égalité valant indépendamment de ce critère naturel.

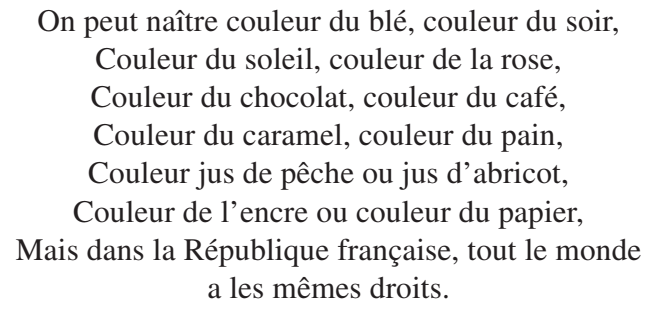

Cette mention de l'égalité des droits annonce dans la version éditée le passage en revue de plusieurs cas d'égalité des droits : «le droit de dormir à l'abri », «le droit d'aller à l'école», «le droit de penser», «le droit au respect» et «le droit de manger». Et pour chaque droit énoncé, sont mis en balance deux types d'individus selon des critères distinctifs respectivement liés à la richesse, à la santé physique, aux croyances et à l'âge. Nous retrouvons ici les droits fondamentaux explorés dans la partie «Liberté». Certains de ces changements, relatifs à l'énoncé de droits, étaient en germe dans l'une des versions intermédiaires du texte profondément remanié que l'on trouve dans le carnet vert (qui, nous le rappelons, est une version postérieure au chemin de fer reproduit ici en figure 8 , puisque plus élaborée dans l'illustration et la mise en page) : le droit d'aller à l'école, le droit de penser, le droit au respect; le droit de manger. Était également présent le droit de dormir, biffé puis remplacé par le droit d'avoir un nom, dont seul le premier sera repris dans la version éditée.

Malgré les variations entre le texte initial et la version éditée, l'auteure a conservé des oppositions : le riche vs le 


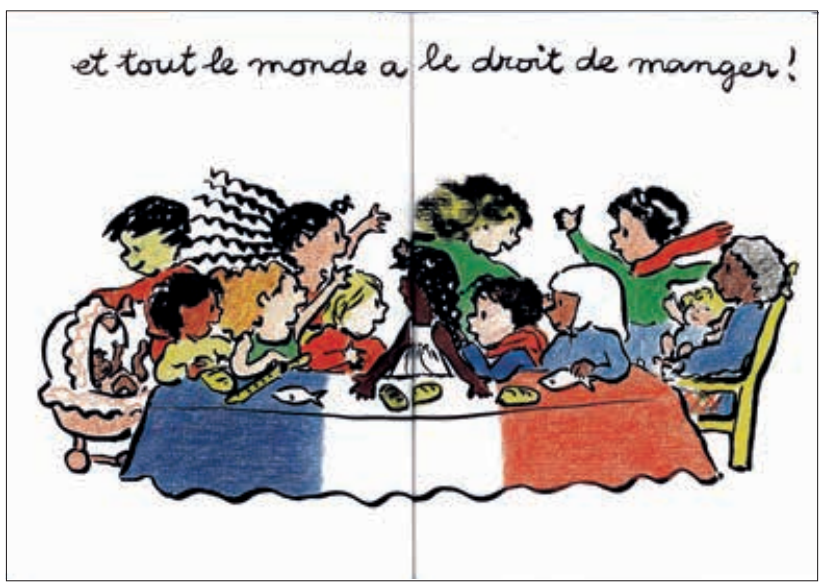

Fig. 9 : Le banquet. Double page (26-27) de la version éditée Agnès Rosenstiehl, Liberté, Égalité, Fraternité, Neuville-sur-Saône, Éditions Chemins de Tr@verse, 2016 - Format : 14,5 cm × $20 \mathrm{~cm}$.

pauvre (opposition relative au droit de dormir à l'abri pour la version éditée et à «qui est le plus une fille?» pour le texte initial); le bébé vs la vieille (opposition relative au droit au respect pour la version éditée et à «qui est le plus chez soi sur terre?» pour le texte initial).

Parmi les modifications les plus significatives, nous pouvons relever dans l'avant-dernière double page, la suppression de tous les «moi, moi, moi» en réponse à la question «Qui est-ce qui a le plus besoin de manger?» au profit d'une grande tablée où chacun profite du partage - un cas significatif où l'illustration vient remplacer le texte (fig. 9); enfin, la dernière double page a été adoucie : un loup qui dévore un mouton, selon l'ordre naturel des choses, est remplacé par un chat chassant une souris (fig. 10)... Ordre naturel respecté, mais cruauté diminuée puisque cette pratique de chasse est plus «familière» et heurtera peutêtre moins le jeune lecteur, même si la cruauté parfois est jubilatoire, quand elle est tenue à distance par la littérature.

Derrière cette étude comparée d'un premier état du texte «Égalité » et de sa version éditée, se donne à lire le souci de l'auteure pour son lectorat : la façon dont l'auteure construit de manière créative le cheminement de l'œuvre, par essais successifs, vient justifier la place du lecteur dans son travail d'écriture : si au départ, elle ne semble l'envisager que comme une abstraction, puisque domine alors, dans ce temps, une centration sur un questionnement créatif, c'est ensuite, après l'exploration de plusieurs «méandres de la Seine » à un moment donné, lorsqu' elle envisage les réponses possibles aux questions qu'elle lui pose, que le lecteur prend possession du texte en l'informant, en le transformant.

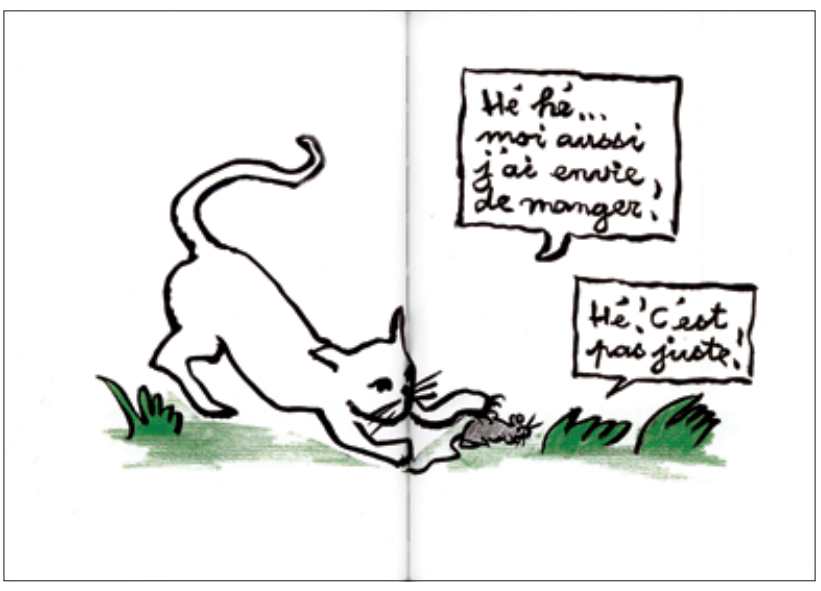

Fig. 10 : Le chat. Double page (28-29) de la version éditée Agnès Rosenstiehl, Liberté, Égalité, Fraternité, Neuville-sur-Saône, Éditions Chemins de Tr@verse, 2016 - Format : 14,5 cm × $20 \mathrm{~cm}$.

\section{Conclusion}

Ce qui fait la particularité, à notre sens, de l'écriture d'Agnès Rosenstiehl, c'est un retour incessant sur le texte visant toujours une efficacité, celle de la simplicité, par épuration successive du texte afin de mettre le jeune lecteur en situation de réfléchir à ce qui lui est présenté, sans qu'aucun discours moralisateur ne lui soit donné. À lui de se faire sa propre idée des comportements à tenir après sa rencontre avec l'œuvre. Et l'illustration, si elle semble seconde (par rapport à la réalisation du canevas de l'œuvre avec la liste des thèmes/questions à traiter, par exemple), participe de cette volonté de faire dire au texte le minimum, tout en en accompagnant le message. Ses états successifs, de l'esquisse à grand trait à la forme finale, viennent parfois modifier le texte.

Ce qui semble motiver une telle pratique de la réécriture, par essais successifs, c'est à la fois la volonté de défendre un projet d'écriture ambitieux (rendre accessibles à de très jeunes enfants des principes fondamentaux essentiels à la vie en société et à l'épanouissement personnel) et le souci du lecteur, qui prend forme dans la transformation même du texte et de l'image. C'est, en effet, par la présence croissante de ce lecteur au fur et à mesure des changements, dans un tâtonnement créatif qui intègre son mode de raisonnement, que le projet d'écriture trouve toute sa force et que le généticien parvient à mesurer modestement ce que signifie la mention «de jeunesse» dans la dénomination de cette littérature destinée au jeune lectorat. 
KARINe Meshoub-ManiÈre est maître de conférences en Sciences du langage à l'ESPE Lille Nord de France où elle assure la formation des futurs professeurs des écoles en français dans le cadre du Master Métiers de l'enseignement, de l'éducation et de la formation. Rattachée au laboratoire CIREL-Théodile (EA4358 Université de Lille), elle mène des recherches en didactique de la grammaire et de l'écriture/réécriture autour de l'exploitation de brouillons d'écrivains jeunesse. Elle a publié dans les revues Repères («Réécrire des textes au cycle 3, repenser les processus d'écriture en master MEEF») et Le Français aujourd'hui («D'une étrange conception de la langue dans quelques manuels scolaires de l'école élémentaire : le cas du "féminin des noms"») et dans l'ouvrage collectif Machines à voler les mots. Idéologies, pratiques et techniques du plagiat, publié aux Éditions universitaires de Dijon.

karine.meshoubmaniere@espe-lnf.fr

\section{Sur les traces de Liberté, Égalité, Fraternité d'Agnès Rosenstiehl}

Écrire pour la jeunesse, Agnès Rosenstiehl, auteure et illustratrice, n'a cessé de l'expérimenter, d'en explorer les contours à travers une centaine de titres publiés à ce jour. Si son personnage de Mimi Cracra - fillette de quatre ans maligne et désobéissante, prête à explorer le monde - l'a rendue célèbre auprès du grand public avec près de 250 épisodes, traduits dans différentes langues, son œuvre n'en demeure pas moins riche et variée. C'est la genèse de l'album Liberté, Égalité, Fraternité, triptyque publié en 1999 au Seuil (réédité en 2016) qui a particulièrement attiré notre attention. Derrière un projet ambitieux - sensibiliser le jeune lectorat (3-6 ans) aux trois termes de la devise républicaine -, une certaine forme de simplicité dans l'écriture et l'illustration apparaît au lecteur adulte et interpelle le généticien. L'analyse de quelques documents de genèse permet ici de mettre au jour la construction de cette simplicité au sein du processus créatif, qui témoigne d'une véritable prise en compte du lecteur au cours de son élaboration.

Agnès Rosenstiehl, author and illustrator, has never stopped experimenting with writing for young people, pushing the limits of the genre through a hundred titles published to date. If Mimi Cracra - a clever and disobedient four-year-old girl, ready to explore the world - is the character who has made her famous with the general public with nearly 250 episodes, translated into different languages, her work remains nevertheless rich and varied. It is the genesis of the picture book Liberté, Égalité, Fraternité, a triptych published in 1999 by Le Seuil (and re-released in 2016), that particularly caught our attention. Behind an ambitious project - to make the young readership (3-6 years old) aware of the three terms of the Republican motto -, a certain form of simplicity in writing and illustration appears to the adult reader and challenges the geneticist. The analysis of some genetic documents reveals the construction of this simplicity within the creative process, which shows that the reader is truly taken into account during its elaboration.

Die Autorin und Illustratorin Agnès Rosenstiehl hörte nie auf, sich im Genre der Jugendliteratur experimentierend zu betätigen und lotete dabei mit bisher etwa hundert veröffentlichten Titeln die Grenzen dieser Literaturgattung aus. Wenngleich vor allem ihre Figur Mimi Cracra - ein bösartiges und ungehorsames vierjähriges Mädchen, das die Welt erforschen will - die Autorin mit fast 250 Episoden, die in verschiedene Sprachen übersetzt wurden, der breiten Öffentlichkeit bekannt machte, ist ihre Arbeit insgesamt vielfältig und abwechslungsreich. Unsere besondere Aufmerksamkeit gilt der Genese von Liberté, Égalité, Fraternité, einem dreiteiligen Werk, das 1999 bei Le Seuil erschien (und 2016 neu herausgegeben wurde). Hinter einem ehrgeizigen Projekt - es will die junge Leserschaft (3-6 Jährige) für die drei Begriffe der republikanischen Devise sensibilisieren - kommt für den erwachsenen Leser eine gewisse Form von Einfachheit in Schrift und Illustration zum Ausdruck und stellt eine Herausforderung für den texgenetischen Forscher dar. Die Analyse einiger genetischer Dokumente erlaubt es, das Erreichen dieser Einfachheit innerhalb des Schaffensprozesses aufzuzeigen, wobei sich eine veritable Rücksichtnahme auf die Leserschaft im Ausarbeitungsprozess zeigt.

Agnès Rosenstiehl, autora e ilustradora, ha experimentado sin cesar la escritura para niños, explorando sus límites a lo largo de una centena de títulos publicados hasta la actualidad. Si ha sido su personaje de Mimi Cracra -niñita de cuatro años pícara y desobediente, dispuesta a explorar el mundo-quien le ha permitido volverse famosa ante el gran público con sus casi 250 episodios traducidos en diferentes idiomas, su obra es no obstante rica y variada. Nos hemos interesado particularmente en la génesis de Liberté, Égalité, Fraternité, tríptico publicado por Seuil en 1999 (y reeditado en 2016). Detrás de este ambicioso proyecto -sensibilizar a los jóvenes lectores de entre tres y seis años a los términos de la divisa republicana-, el lector adulto percibe una cierta forma de simplicidad en la escritura y la ilustración, que intriga al geneticista. El análisis de algunos documentos de génesis nos permitirá aquí echar luz sobre la construcción de esta simplicidad en el seno del proceso creativo, que muestra como, a lo largo de su elaboración, el lector es tomado cabalmente en cuenta.

A autora e ilustradora Agnès Rosenstiehl nunca cessou de experimentar com a escrita de livros infantis, de que tem cem títulos publicados até à data. A personagem de Mimi Cracra - menina de quatro anos marota e desobediente, pronta para explorar o mundo - tornou-a famosa com cerca de 250 episódios, traduzidos em diferentes línguas. Mas o seu restante trabalho é não menos rico e variado. A génese do album Liberté, Égalité, Fraternité, tríptico publicado pela Seuil em 1999 (e reeditado em 2016), parte de um projeto ambicioso - sensibilizar jovens leitores de 3-6 anos para os três termos da divisa republicana. Mas o que mais chama a atenção do leitor adulto e interpela o geneticista, é uma certa forma de simplicidade na escrita e na ilustração que, como revela a análise de alguns documentos genéticos, é uma simplicidade construída no decurso do processo creativo, elaborada a pensar no leitor.

Agnès Rosenstiehl, autrice e illustratrice, non ha mai smesso di sperimentare e di esplorare i limiti della scrittura per l'infanzia, attraverso un centinaio di titoli pubblicati fino ad oggi. Se il suo personaggio di Mimi Cracra - bambina di quattro anni furba e disobbediente, pronta ad esplorare il mondo - l'ha resa .../... 
.../... celebre presso un vasto pubblico, grazie ai circa 250 episodi pubblicati e tradotti in diverse lingue, la sua opera rimane ricca e varia. La nostra attenzione è stata attratta particolarmente dalla genesi dell'album Liberté, Égalité, Fraternité, trittico pubblicato da Seuil nel 1999 (e riedito nel 2016). Dietro un ambizioso progetto - sensibilizzare i giovani lettori (dai tre ai sei anni) ai tre concetti del motto repubblicano -, appare una certa forma di semplicità nella scrittura e nell'illustrazione, che interroga l'adulto e il ricercatore. L'analisi di alcuni documenti genetici mette in luce la costruzione di questa semplicità al centro del processo creativo, che testimonia, nel corso della sua elaborazione, di una reale considerazione del lettore. 